\title{
DCE-MRI is more sensitive than IVIM-DWI for assessing anti-angiogenic treatment- induced changes in colorectal liver metastases
}

Mihaela Rata ${ }^{1,2,9^{*}}$, Khurum Khan ${ }^{3}$, David J Collins ${ }^{1,2}$, Dow-Mu Koh ${ }^{1,2}$, Nina Tunariu ${ }^{1,2}$, Maria Antonietta Bali, ${ }^{1,2}$ James $d^{\prime}$ Arcy $^{2,4}$, Jessica M Winfield ${ }^{1,2}$, Simona Picchia ${ }^{1}$, Nicola Valeri ${ }^{3,5,6}$, lan Chau ${ }^{3}$, David Cunningham ${ }^{3}$, Matteo Fassan ${ }^{7,8}$, Martin O Leach ${ }^{1,2}$ and Matthew R Orton ${ }^{1,2}$

\begin{abstract}
Background: Diffusion weighted imaging (DWI) with intravoxel incoherent motion (IVIM) modelling can inform on tissue perfusion without exogenous contrast administration. Dynamic-contrast-enhanced (DCE) MRI can also characterise tissue perfusion, but requires a bolus injection of a Gadolinium-based contrast agent.

This study compares the use of DCE-MRI and IVIM-DWI methods in assessing response to anti-angiogenic treatment in patients with colorectal liver metastases in a cohort with confirmed treatment response.

Methods: This prospective imaging study enrolled 25 participants with colorectal liver metastases to receive Regorafenib treatment. A target metastasis $>2 \mathrm{~cm}$ in each patient was imaged before and at 15 days after treatment on a 1.5T MR scanner using slice-matched IVIM-DWI and DCE-MRI protocols.

MRI data were motion-corrected and tumour volumes of interest drawn on $b=900 \mathrm{~s} / \mathrm{mm}^{2}$ diffusion-weighted images were transferred to DCE-MRI data for further analysis. The median value of four IVIM-DWI parameters [diffusion coefficient $D\left(10^{-3} \mathrm{~mm}^{2} / \mathrm{s}\right)$, perfusion fraction $\mathrm{f}(\mathrm{ml} / \mathrm{ml})$, pseudodiffusion coefficient $\mathrm{D}^{*}\left(10^{-3} \mathrm{~mm}^{2} / \mathrm{s}\right)$, and their product $\mathrm{fD}^{*}\left(\mathrm{~mm}^{2} / \mathrm{s}\right)$ ] and three DCE-MRI parameters [volume transfer constant $\mathrm{K}^{\text {trans }}\left(\mathrm{min}^{-1}\right)$, enhancement fraction $\mathrm{EF}(\%)$, and their product $\left.\mathrm{KEF}\left(\mathrm{min}^{-1}\right)\right]$ were recorded at each visit, before and after treatment.

Changes in pre- and post-treatment measurements of all MR parameters were assessed using Wilcoxon signed-rank tests $(P<0.05$ was considered significant). DCE-MRI and IVIM-DWI parameter correlations were evaluated with Spearman rank tests.

Functional MR parameters were also compared against Response Evaluation Criteria In Solid Tumours v.1.1 (RECIST) evaluations.
\end{abstract}

\footnotetext{
* Correspondence: Mihaela.Rata@icr.ac.uk

'Department of Radiology, MRI Unit, The Royal Marsden NHS Foundation Trust, London, United Kingdom

${ }^{2}$ Division of Radiotherapy and Imaging, The Institute of Cancer Research, London, United Kingdom

Full list of author information is available at the end of the article
}

(C) The Author(s). 2021 Open Access This article is licensed under a Creative Commons Attribution 4.0 International License, which permits use, sharing, adaptation, distribution and reproduction in any medium or format, as long as you give appropriate credit to the original author(s) and the source, provide a link to the Creative Commons licence, and indicate if changes were made. The images or other third party material in this article are included in the article's Creative Commons licence, unless indicated otherwise in a credit line to the material. If material is not included in the article's Creative Commons licence and your intended use is not permitted by statutory regulation or exceeds the permitted use, you will need to obtain permission directly from the copyright holder. To view a copy of this licence, visit http://creativecommons.org/licenses/by/4.0/ The Creative Commons Public Domain Dedication waiver (http://creativecommons.org/publicdomain/zero/1.0/) applies to the data made available in this article, unless otherwise stated in a credit line to the data. 
Results: Significant treatment-induced reductions of DCE-MRI parameters across the cohort were observed for EF ( 91.2 to $50.8 \%, P<0.001)$, KEF (0.095 to $\left.0.045 \mathrm{~min}^{-1}, P<0.001\right)$ and $K^{\text {trans }}\left(0.109\right.$ to $\left.0.078 \mathrm{~min}^{-1}, P=0.002\right)$. For IVIM-DWI, only D (a non-perfusion parameter) increased significantly post treatment $\left(0.83\right.$ to $\left.0.97 \times 10^{-3} \mathrm{~mm}^{2} / \mathrm{s}, P<0.001\right)$, while perfusion-related parameters showed no change. No strong correlations were found between DCE-MRI and IVIM-DWI parameters. A moderate correlation was found, after treatment, between $K^{\text {trans }}$ and $D^{*}(r=0.60 ; P=0.002)$ and $\mathrm{fD}^{*}(r=0.67 ; P<0.001)$. When compared to RECIST v.1.1 evaluations, KEF and D correctly identified most clinical responders, whilst non-responders were incorrectly identified.

Conclusion: IVIM-DWI perfusion-related parameters showed limited sensitivity to the anti-angiogenic effects of Regorafenib treatment in colorectal liver metastases and showed low correlation with DCE-MRI parameters, despite profound and significant post-treatment reductions in DCE-MRI measurements.

Trial registration: NCT03010722 clinicaltrials.gov; registration date $6^{\text {th }}$ January 2015.

Keywords: Dynamic contrast enhanced MRI (DCE-MRI), Intravoxel incoherent motion diffusion weighted imaging (IVIM-DWI), Perfusion, Colorectal liver metastasis, Clinical trial.

\section{Background}

Regorafenib (Stivarga ${ }^{\circ}$ ), a small molecule multiple kinase inhibitor with action against pro-angiogenic and proproliferative targets, has been shown to prolong disease survival for colorectal cancer patients [1]. However, treatment using Regorafenib is associated with side effects in up to $98 \%$ of patients [2]. Hence, early identification of patients who are benefiting from treatment can help to individualize treatment, by potentially terminating ineffective treatments and minimizing drug toxicity.

Functional MRI techniques, such as dynamic contrast enhanced (DCE)-MRI and diffusion weighted imaging (DWI), provide information about perfusion and cellularity. DCEMRI can characterise tissue vascularisation [3, 4], but requires repeated rapid image acquisition of the tumour, sustained over a few minutes after bolus injection of a Gadolinium-based contrast agent. The temporal evolution of enhancement on T1-weighted imaging is used to model and derive quantitative vascular parameters. Studies have shown that DCE-MRI parameters can provide insights into early therapeutic effects or disease outcome [5]. In colorectal liver metastases treated with Regorafenib, an early reduction in KEF (product of transfer constant $\mathrm{K}^{\text {trans }}$ and enhancing fraction EF) value by $>70 \%$ was associated with better disease control [6].

Diffusion-weighted imaging provides insights into tissue water mobility and does not require exogenous contrast injection. By performing DWI using multiple bvalues combined with intravoxel incoherent motion (IVIM) modelling, tissue water diffusion is expected to be separated from pseudo-diffusion that can reflect tissue perfusion [7, 8]. IVIM-DWI perfusion measurements can help characterization and assess response to anticancer treatments [9]. However, clinical IVIM-DWI studies [10-12] in colorectal liver metastases have been inconclusive about the value of the perfusion-sensitive IVIM parameters for assessing drug therapeutic effects. This may be related to the fact that colorectal liver metastases are predominantly hypovascular and their IVIM-DWI derived parameters have poor measurement repeatability [13], which can limit the sensitivity of the technique in detecting vascular changes.

Previous studies compared measurements of vascular parameters using DCE-MRI with IVIM-DWI in rectal cancer $[14,15]$. A similar assessment of the two techniques in colorectal liver metastases will aid understanding the relative merits of both techniques in their deployment in clinical practice. Hence, the aim of this study is to compare the use of DCE-MRI and IVIMDWI techniques in assessing the response to Regorafenib treatment in colorectal liver metastases. Note that this work follows up a clinical report of the efficacy of drug treatment with Regorafenib [6]. In the present study, we compare IVIM-DWI measurements (previously not reported) against DCE-MRI measurements.

\section{Methods}

\section{Study population}

The trial recruitment was approved by our institutional review board (National Research Ethics Service, NRES Committee London-Fulham; Research Ethics Committee reference 14/LO/1812) and informed written consent was obtained from each patient.

This prospective phase II study (NCT03010722 registry number on clinicaltrials.gov) enrolled 25 patients with colorectal liver metastases, between March 2015 and May 2016, to a single drug Regorafenib treatment. The three inclusion criteria were: (1) patients at least $18 \mathrm{y}$-old with a WHO performance status of $0-1$ for which all conventional treatments were exhausted; (2) patients had metastatic disease amenable to biopsy and repeat measurements with DCE-MRI; (3) patients were confirmed to have a RAS-mutant cancer type. The 
patient cohort, including case exclusions, is presented in Fig. 1.

In each patient, a target metastasis $(>2 \mathrm{~cm})$ was selected to undergo DCE-MRI and IVIM-DWI before (day -7 to 0 ) and at day 15 after treatment. Five additional patients with liver metastases were recruited (February to June 2017) to assess same-day test-retest repeatability of the IVIM-DWI technique used in the prospective study.

\section{MR Image acquisition}

All imaging was performed on a $1.5 \mathrm{~T}$ MR scanner (MAGNETOM Avanto, Siemens Healthcare, Erlangen, Germany), see full parameters in Table 1. The IVIMDWI scans were performed prior to DCE-MRI studies to avoid possible effects of gadolinium contrast on the IVIM-DWI measurements.

IVIM-DWI protocol. Free-breathing coronal IVIMDWI was acquired using a 2D echo planar imaging sequence with a 3 direction scan trace method and 8 bvalues [range $0-900 \mathrm{~s} / \mathrm{mm}^{2}$ ]. DWI for each gradient direction was obtained and 5 independent acquisitions (no averaging) were acquired over $10 \mathrm{~min}$.
DCE-MRI protocol. A standard dose of contrast agent (Dotarem, $0.2 \mathrm{ml} / \mathrm{kg}$ ) followed by $20 \mathrm{ml}$ of saline were delivered by an automatic power injector at $3 \mathrm{ml} / \mathrm{s}$. Breath-hold coronal DCE-MRI data were acquired using a 3D spoiled gradient echo sequence (VIBE-volumetric interpolated breath-hold examination) that matched the field of view and resolution of IVIM-DWI. Dynamic scans were preceded by a calibration scan with the same parameters, but at a lower flip angle $\left(2^{\circ}\right)$ and with 7 averages, to enable contrast quantification [16]. Patients were imaged using a sequential breath-hold technique optimised for liver lesions [17]: two imaging volumes were acquired during each $6 \mathrm{~s}$ breath-hold, followed by a $6 \mathrm{~s}$ breathing gap; a total of 40 volumes were acquired over $4 \mathrm{~min}$.

\section{MRI data processing}

MRI data were motion-corrected (2D techniques, Matlab 2017a, see Supplementary material), and tumour volumes of interest (VOI) were drawn by an experienced radiologist on high-b-value IVIM-DW images (b900) for analysis, see Fig. 2. The VOIs were transferred to the

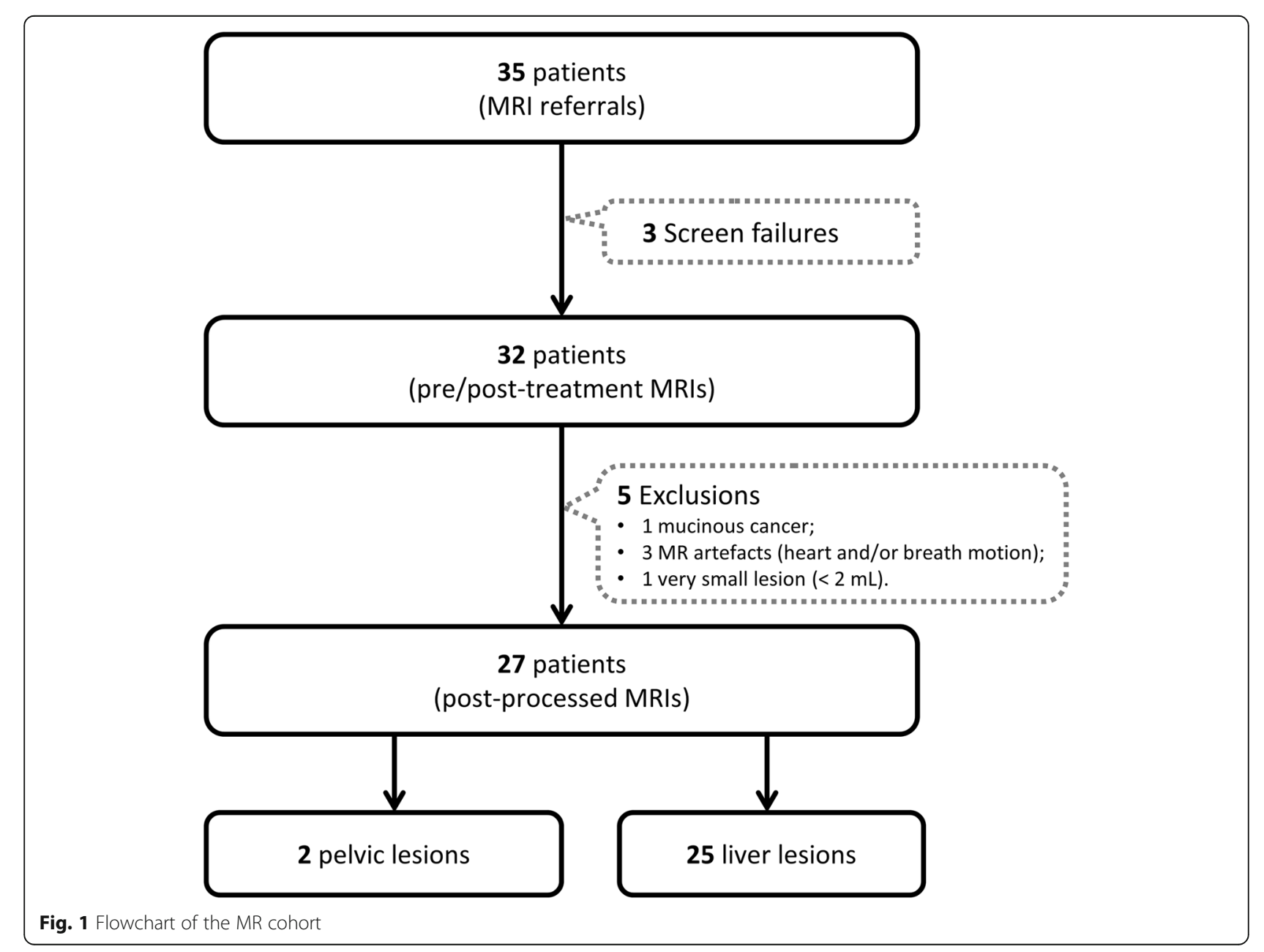


Table 1 MR parameters for the IVIM-DWI and DCE-MRI sequences.

\begin{tabular}{|c|c|c|}
\hline \multirow[t]{2}{*}{ MRI Parameters } & \multicolumn{2}{|c|}{ Liver tumours ( $n=25$ patients) } \\
\hline & IVIM-DWI & DCE-MRI \\
\hline Sequence & 2D single shot echo planar imaging & 3D gradient echo \\
\hline Acquisition plane & Coronal & Coronal \\
\hline Breathing mode & Free breathing & Breath holding \\
\hline Total acquisition time [min:s] & $10: 25$ & 04:18 \\
\hline Time per single acquisition [min:s] & 02:05 & 04:18 \\
\hline Number of averages & 5 & 1 \\
\hline Acquired voxel size $\left[\mathrm{mm}^{3}\right]$ & $3.1 \times 3.1 \times 5$ & $3.1 \times 3.1 \times 5$ \\
\hline Reconstructed voxel size $\left[\mathrm{mm}^{3}\right]$ & $1.56 \times 1.56 \times 5$ & $1.56 \times 1.56 \times 5$ \\
\hline Slice thickness [mm] & 5 & 5 \\
\hline $\mathrm{TR}[\mathrm{ms}]$ & 5000 & 3 \\
\hline TE [ms] & 60 & 0.89 \\
\hline Flip angle $\left[{ }^{\circ}\right]$ & - & 11 \\
\hline Slices per slab & 20 & 14 \\
\hline Slice gap [mm] & 0 & 0 \\
\hline Slice oversampling [\%] & - & 14.3 \\
\hline Matrix (FE x PE) & $128 \times 128$ & $128 \times 128$ \\
\hline $\mathrm{FOV}\left[\mathrm{mm}^{2}\right]$ & $400 \times 400$ & $400 \times 400$ \\
\hline Dynamic measurements & - & 40 \\
\hline Breath holding (pause $6 \mathrm{~s}$ after dyn2, dyn4,etc.) & no & yes \\
\hline Receiver bandwidth [Hz/Pixel] & 1860 & 650 \\
\hline $\begin{array}{l}\text { Parallel acquisition (GRAPPA) } \\
\text { (PE acceleration factor } x \text { reference lines) }\end{array}$ & $2 \times 30$ & $2 \times 24$ \\
\hline Phase partial Fourier & $7 / 8$ & no \\
\hline Slice partial Fourier & - & $6 / 8$ \\
\hline Fat suppression & SPAIR & none \\
\hline 8 b-values $\left[\mathrm{s} / \mathrm{mm}^{2}\right]$ & $0,20,40,60,120,240,480,900$ & - \\
\hline Diffusion times [ms] & $\delta=14.6 ; \Delta=24$ & - \\
\hline
\end{tabular}

$T R=$ repetition time; $T E=e c h o$ time

$F E=$ frequency encoding; $P E=$ phase encoding; $F O V=$ field of view

GRAPPA=GeneRalized Autocalibrating Partial Parallel Acquisition

$S P A I R=S P e c t r a l$ Attenuated Inversion Recovery

DCE-MR images for quantitative analysis. Voxel-wise analysis of the delineated VOI was performed using an in-house software designed for each imaging technique, and median values of the parameters of interest for both IVIM-DWI and DCE-MRI were reported before and after treatment for every patient.

IVIM-DWI data. A model using a Markov random field approach generated IVIM-DWI estimates using a Matlab script (Matlab 2017a; see Supplementary material). The four IVIM-DWI parameters were: diffusion coefficient $D$, perfusion fraction $f$ (proportion of a voxel volume occupied by capillaries), pseudodiffusion coefficient $\mathrm{D}^{*}$ (convective motion of blood in vessel network), and their product $\mathrm{fD}^{*}$.

DCE-MRI data. The pharmacokinetic analysis used the extended Kety/Tofts model $[18,19]$ in conjunction with a cosine-based arterial input function model [20] derived from population-averaged values [21] and was applied using a software from [22].The following main DCE-MRI parameters were reported: volume transfer constant between plasma and extracellular extravascular space $\left(\mathrm{K}^{\text {trans }}\right)$ and the enhancement fraction (EF). The EF was defined as percentage of voxels within the VOI that enhance above the noise floor. A voxel was considered to be enhanced if its post-contrast signal intensity was at least one standard deviation above the mean pre-contrast signal for a period of $60 \mathrm{~s}$ after the arrival of contrast in the lesion.

Parameters were computed to account for additional necrosis after treatment, which typically manifests by non-enhancement. Two tumour-derived $K^{\text {trans }}$ estimates were reported: the whole tumour $\mathrm{K}^{\text {trans }}($ all), and valid voxels only $K^{\text {trans }}$ (nonzeros), i.e. excluding all non- 

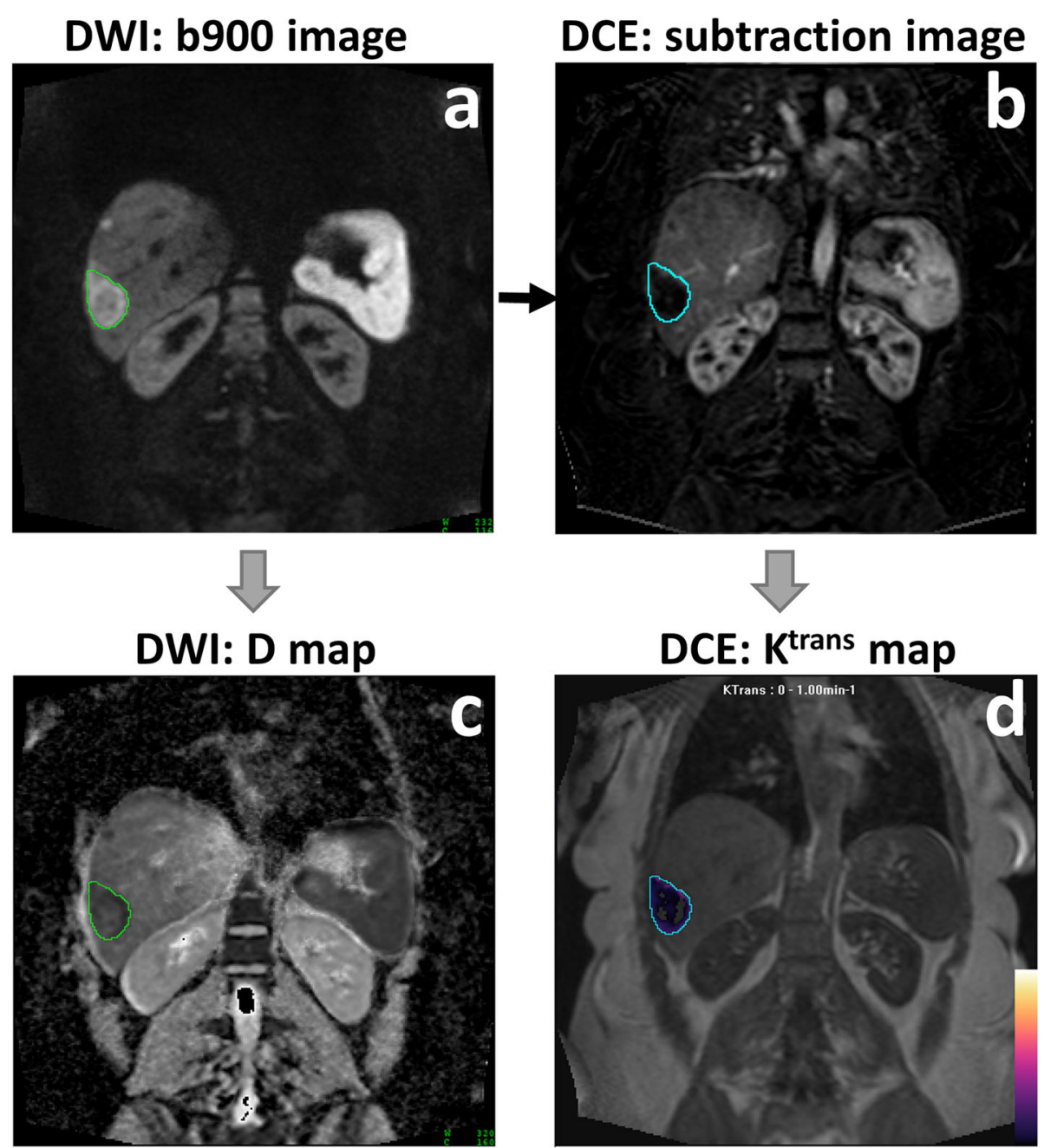

Fig. 2 VOl-based data processing (example of one slice out of the 8 evaluated) for a 48 year old female patient with a lesion in segments $7 / 8$ of the liver. VOI was drawn on the highest-b-value image (a), then transferred to the DCE-MRI subtraction image (b). The subtraction image was calculated as the difference between the dynamic image with peak enhancement within the liver parenchyma (dynamic 9/40) and the first pre-contrast image (dynamic 1/40). The VOls were used in conjunction with each of the computed maps to derive the median values of parameters of interest: $\mathrm{D}$ (c) and $K^{\text {trans }}(\mathbf{d})$ are shown here. Note that $K^{\text {trans }}$ map is shown overlaid on the last dynamic image of the DCE-MRI acquisition (dynamic 40/40)

enhancing voxels. Potential change in the volume of enhancing tumour after treatment (such as new necrosis) was accounted for by reporting a new parameter which incorporates both effects: $\mathrm{KEF}=\mathrm{K}^{\text {trans }}$ (nonzeros) $\mathrm{x} \mathrm{EF}$ [23]. Note that this parameter is the product of the summarized median values of its two components, and does not rely on a direct voxel-wise approach.

\section{Statistical analysis}

Treatment-induced changes on all parameters were assessed with Wilcoxon signed-rank tests as parameters were not normally distributed. Correlations between DCE-MRI ( $\left.{ }^{\text {trans }}, \mathrm{KEF}\right)$ and IVIM-DWI (f, $\left.\mathrm{D}^{*}, \mathrm{fD}^{*}\right)$ parameters were assessed by Spearman rank tests. The IVIM-DWI repeatability was assessed with Bland-
Altman analysis. For all analyses, a P-value of $<0.05$ was deemed statistically significant.

\section{Results}

\section{Patient demographics}

All 25 study patients (16 men, 9 women; mean age $64.4 \pm 10.7$ years) successfully completed all imaging studies and their clinical characteristics are presented in Table 2. Another 5 patients ( 3 men, 2 women; mean age $61.0 \pm 11.6$ years) underwent short term test-retest repeatability studies for the IVIM-DWI method only (Table 2).

DCE-MRI and IVIM-DWI assessment of treatment response The treatment response of the whole study cohort, as measured by DCE-MRI and IVIM-DWI, is shown in 
Table 2 Clinical characteristics of patients for the two cohorts

\begin{tabular}{|c|c|}
\hline \multicolumn{2}{|c|}{$\begin{array}{l}\text { Main cohort ( } N=25) \text { : phase II clinical trial patients; DCE-MRI vs. } \\
\text { IVIM-DWI }\end{array}$} \\
\hline Disease & liver metastases from colorectal cancer \\
\hline Primary cancer & colorectal (all patients) \\
\hline Treatment & $\begin{array}{l}\text { oral anti-angiogenic drug (Regorafenib) } \\
\text { administered daily }\end{array}$ \\
\hline Sex [Female/Male] & $9 / 16$ \\
\hline $\begin{array}{l}\text { Age in years (mean } \pm S D \text {; } \\
\text { range) }\end{array}$ & $64.4 \pm 10.7 ; 44-86$ \\
\hline $\begin{array}{l}\text { Lesion volume in } \mathrm{mL} \text { (mean } \\
\pm \mathrm{SD} \text {; range) }\end{array}$ & $45.8 \pm 60.1 ; 2.2-265.1$ \\
\hline \multicolumn{2}{|c|}{ Repeatability cohort $(N=5)$ : clinical patients; IVIM-DWI } \\
\hline Disease & $\begin{array}{l}\text { liver metastases from gastrointestinal } \\
\text { cancer }\end{array}$ \\
\hline Primary cancer & 3 colorectal, 1 stomach, 1 caecal \\
\hline Treatment & not relevant \\
\hline Sex [Female/Male] & $2 / 3$ \\
\hline $\begin{array}{l}\text { Age in years (mean } \pm S D \text {, } \\
\text { range) }\end{array}$ & $61.0 \pm 11.6 ; 50-80$ \\
\hline $\begin{array}{l}\text { Lesion volume in } \mathrm{mL} \text { (mean } \\
\pm \mathrm{SD} \text {, range) }\end{array}$ & $91.9 \pm 164.5 ; 4.1-385.6$ \\
\hline
\end{tabular}

$S D=$ standard deviation

Fig. 3 using box plots overlaid with ladder plots for individual patients. Significant treatment-induced reductions of the perfusion-related DCE-MRI parameters were observed for EF ( $91.2 \%$ vs.50.8\%, $P<0.001)$, KEF (0.095 vs.
0.045, $P<0.001)$ and $K^{\text {trans }}\left(0.109\right.$ vs. $0.078 \mathrm{~min}^{-1}, P=$ 0.002). A typical example of MR parametric maps from a 59 year-old man with liver metastasis is shown in Fig. 4, demonstrating a decrease in $K^{\text {trans }}$ and EF after treatment.

For IVIM-DWI, only the diffusion parameter, D, increased significantly post treatment $\left(0.83\right.$ vs. $0.97 \times 10^{-3}$ $\left.\mathrm{mm}^{2} / \mathrm{s}, P<0.001\right)$, in keeping with reducing cellularity. However, the perfusion-related parameter changes (f, $D^{*}$ and $\mathrm{fD}^{*}$ ) did not reach statistical significance. The detailed results including P-values of the pre/post-treatment Wilcoxon test of all MR parameters are presented in Table 3.

\section{Correlation of DCE-MRI and IVIM-DWI parameters}

Scatter plots for the IVIM-DWI perfusion parameters $\left(D^{*}\right.$, $f$ and $\mathrm{fD}^{*}$ ) versus the DCE-MRI parameter $\mathrm{K}^{\text {trans }}$ are presented in Fig. 5. Spearman correlation tests found no strong (i.e. correlation coefficient $r>0.7$ ) correlation between $\mathrm{K}^{\text {trans }}$ and IVIM-DWI parameters. A moderate correlation $(0.5<\mathrm{r}<0.7)$ was found, after treatment, between $K^{\text {trans }}$ and two perfusion parameters: $D^{*}(r=0.60 ; P=0.002)$ and $\mathrm{fD}^{*}(r=0.67 ; P<0.001)$. No significant correlation was found between $\mathrm{f}$ and $\mathrm{K}^{\text {trans }}$ at any timepoint during treatment.

\section{Repeatability of IVIM-DWI parameters}

Individual Bland Altman plots for each IVIM-DWI parameter as derived from the five patient cohort are

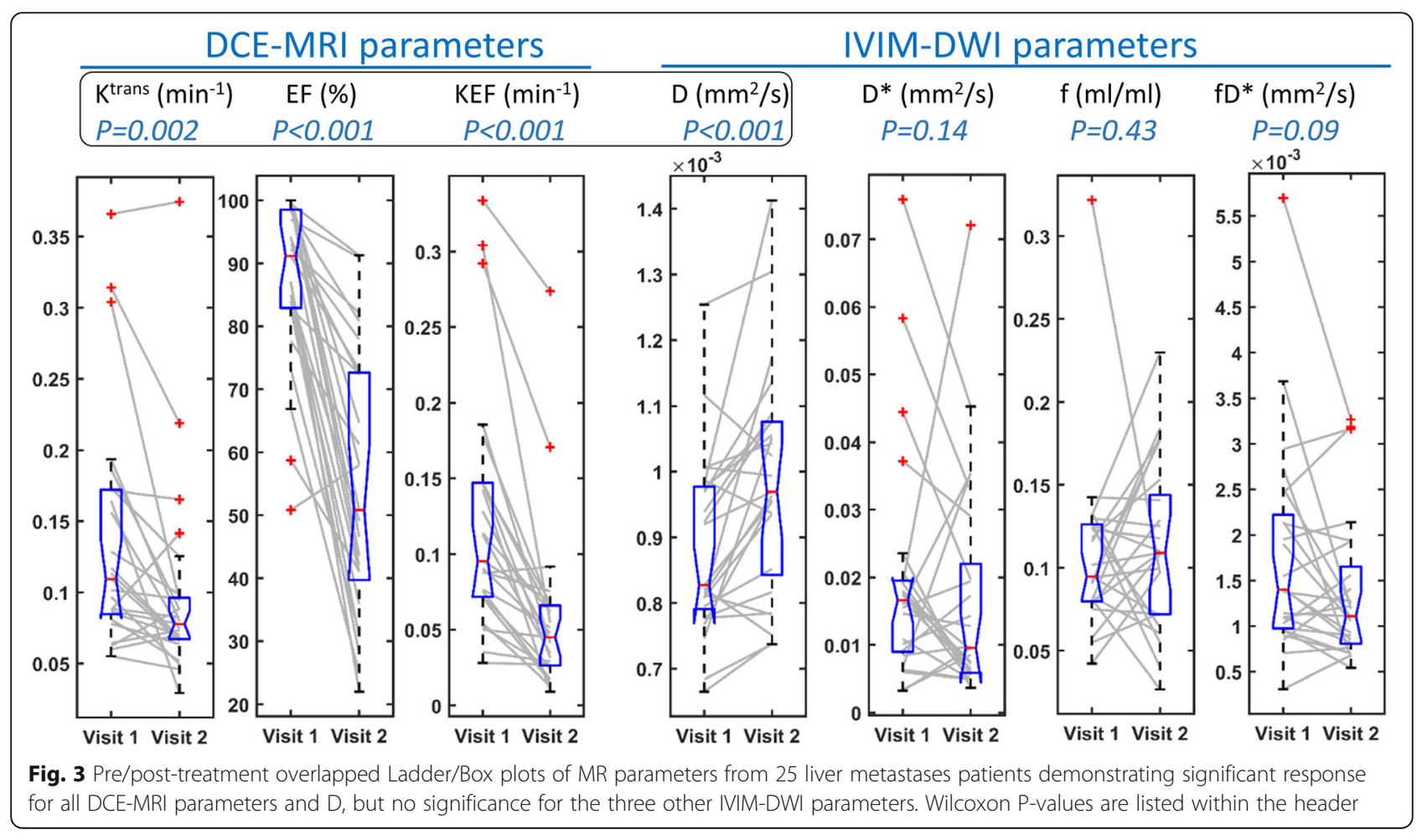




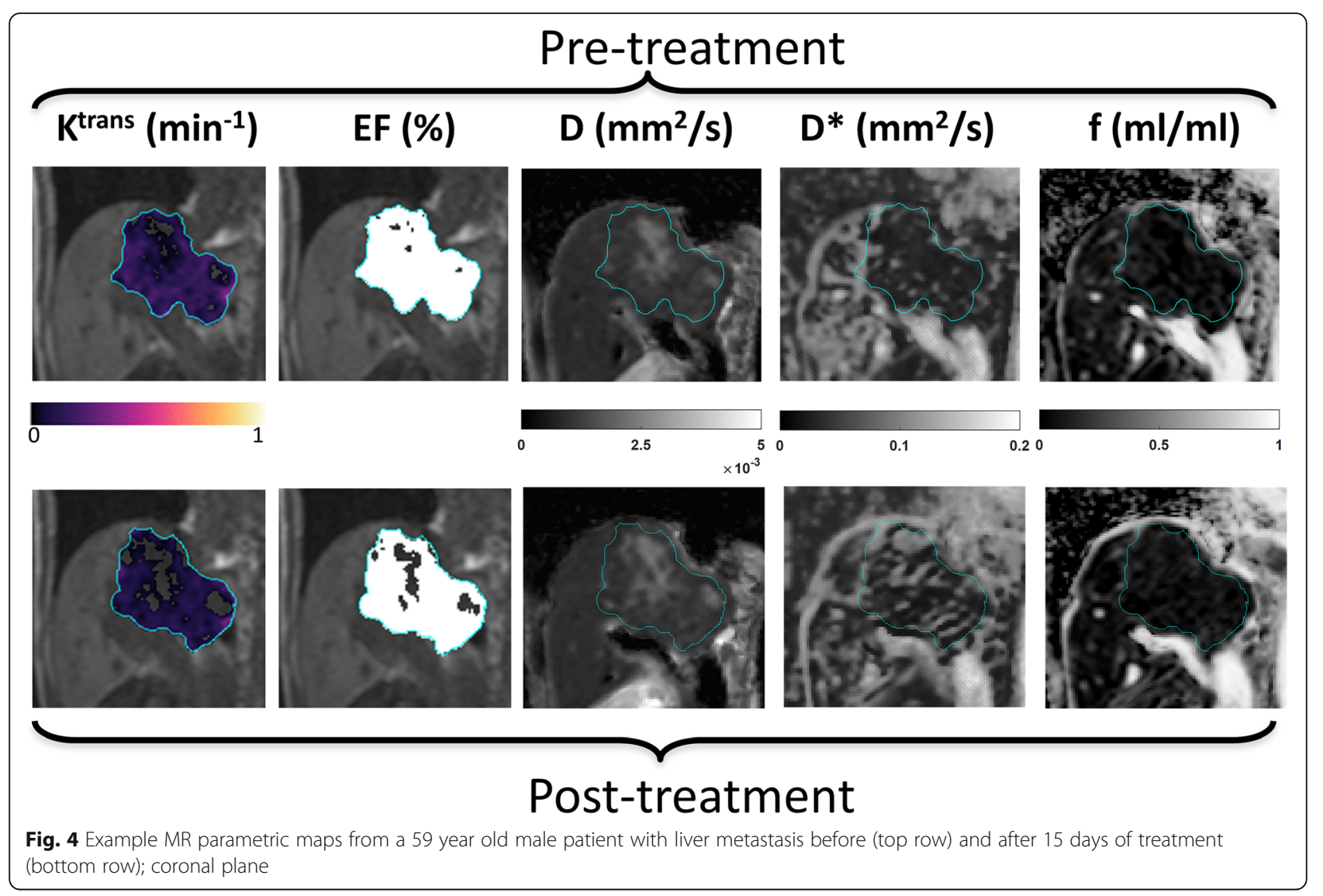

presented in Fig. 6. The IVIM-DWI parameter repeatability was lowest for $\mathrm{D}$ (coefficient of variation $\mathrm{CV}=$ $2.9 \%)$, followed by $\mathrm{f}(\mathrm{CV}=48 \%), \mathrm{D}^{*}(\mathrm{CV}=60 \%)$ and $\mathrm{fD}^{*}$ $(\mathrm{CV}=117 \%)$. Full statistical results are presented in Table 3. The perfusion-sensitive parameters of colorectal liver metastases were considerably less repeatable compared with tissue diffusivity measurement (D).
Correlation of MR parameters and RECIST

Figure 7 presents waterfall plots of the four MR parameters showing statistical significance in assessing early tumour response at day 15 post-treatment: three DCEMRI parameters ( $\mathrm{K}^{\text {trans }}, \mathrm{EF}$ and KEF) and the nonperfusion D parameter derived from IVIM-DWI. Note that the disease control rate, measured by Response

Table 3 Median values and main statistics of the DCE-MRI and IVIM-DWI parameters (Wilcoxon test; paired t-test and Bland-Altman analysis). Values with the \# symbol were statistically significant at $P<0.05$.

\begin{tabular}{|c|c|c|c|c|c|c|c|}
\hline Main cohort $(n=25)$ & $\mathrm{K}^{\text {trans }}(\mathrm{nz})$ & EF & KEF & D & $\mathrm{D}^{*}$ & $f$ & $f D^{*}$ \\
\hline Median value [Visit 1] & 0.109 & 91.2 & 0.095 & 0.00083 & 0.0166 & 0.0947 & 0.0014 \\
\hline Median value [Visit 2] & 0.078 & 50.8 & 0.045 & 0.00097 & 0.0095 & 0.1091 & 0.0011 \\
\hline$P$ value (Wilcoxon signed-rank test) & $0.002^{\#}$ & $<0.001^{\#}$ & $<0.001^{\#}$ & $<0.001^{\#}$ & 0.14 & 0.43 & 0.09 \\
\hline IVIM Repeatability cohort $(n=5)$ & $K^{\text {trans }}(n z)$ & EF & KEF & D & $D^{*}$ & $f$ & $f D^{*}$ \\
\hline Mean value [Repeat 1] & $x$ & $x$ & $x$ & 0.0012 & 0.0348 & 0.0885 & 0.0027 \\
\hline Mean value [Repeat 2] & $x$ & $x$ & $x$ & 0.0012 & 0.0404 & 0.1065 & 0.0045 \\
\hline$P$ value (paired t-test) & & & & 0.57 & 0.61 & 0.44 & 0.41 \\
\hline \multicolumn{8}{|l|}{ Bland-Altman tests } \\
\hline standard deviation (SD) & & & & 0.00003 & 0.022 & 0.047 & 0.004 \\
\hline mean bias & & & & -0.00001 & 0.006 & 0.018 & 0.002 \\
\hline coefficient of variation (CV) \%; SD/mean & & & & 2.9 & 60 & 48 & 117 \\
\hline
\end{tabular}




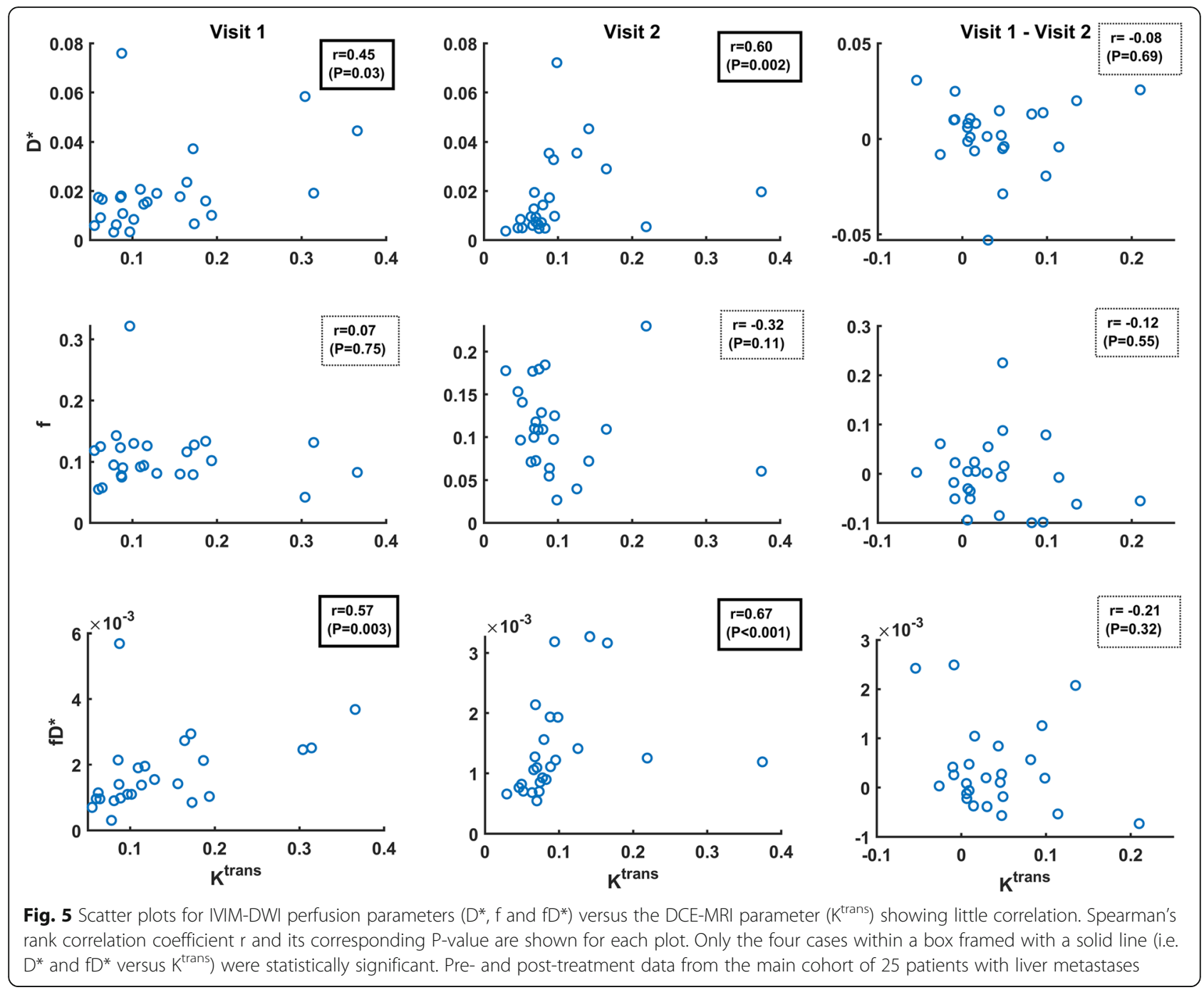

Evaluation Criteria In Solid Tumors v.1.1 (RECIST), was obtained at a later timepoint (week 8 post-treatment) and covered 21/25 patients as four patients were not evaluated by RECIST. Out of the 21 patients with RECIST v.1.1 evaluations available, KEF correctly identified 12/13 clinical responders and incorrectly identified 8/8 non-responders, while D correctly identified 11/13 responders and incorrectly identified $6 / 8$ nonresponders.

\section{Discussion}

This prospective single-center study was performed in 25 patients with colorectal liver metastases treated with single-agent Regorafenib (anti-angiogenic and antiproliferative effects). Across the cohort, there was a significant response measured by DCE-MRI demonstrating an overall decrease in the median $\mathrm{K}^{\text {trans }}$, EF and KEF values after treatment. This early decrease of DCE-MRI parameters was correlated, at later timepoints, with standard clinical biomarkers, including the endothelial marker CD31 which confirmed the anti-angiogenic effect of the drug in a smaller subcohort [6].

Additionally, the statistically significant functional MR parameters $\left(\mathrm{K}^{\text {trans }}, \mathrm{EF}, \mathrm{KEF}\right.$ and $\left.\mathrm{D}\right)$ showed a good correlation with RECIST v.1.1 measurements (Fig. 7). Both KEF and D parameters correctly identified most clinical responders (12/13 and $11 / 13$, respectively), whilst incorrectly classifying most non-responders as responders. Note that MR measurements were performed 2 weeks post treatment and the RECIST v.1.1 evaluation after 8 weeks. Such a discrepancy limits the interpretation of these comparisons since the lesion behaviour over time cannot be fully assessed (e.g. an early responder at 2 weeks might translate into a non-responder at a later timepoint).

In this study, we investigated whether perfusionrelated IVIM-DWI effects mirror the perfusion effects measured using DCE-MRI in a cohort treated uniformly with a drug that has anti-angiogenic effects. To ensure optimum comparison, both DCE-MRI and IVIM-DWI 


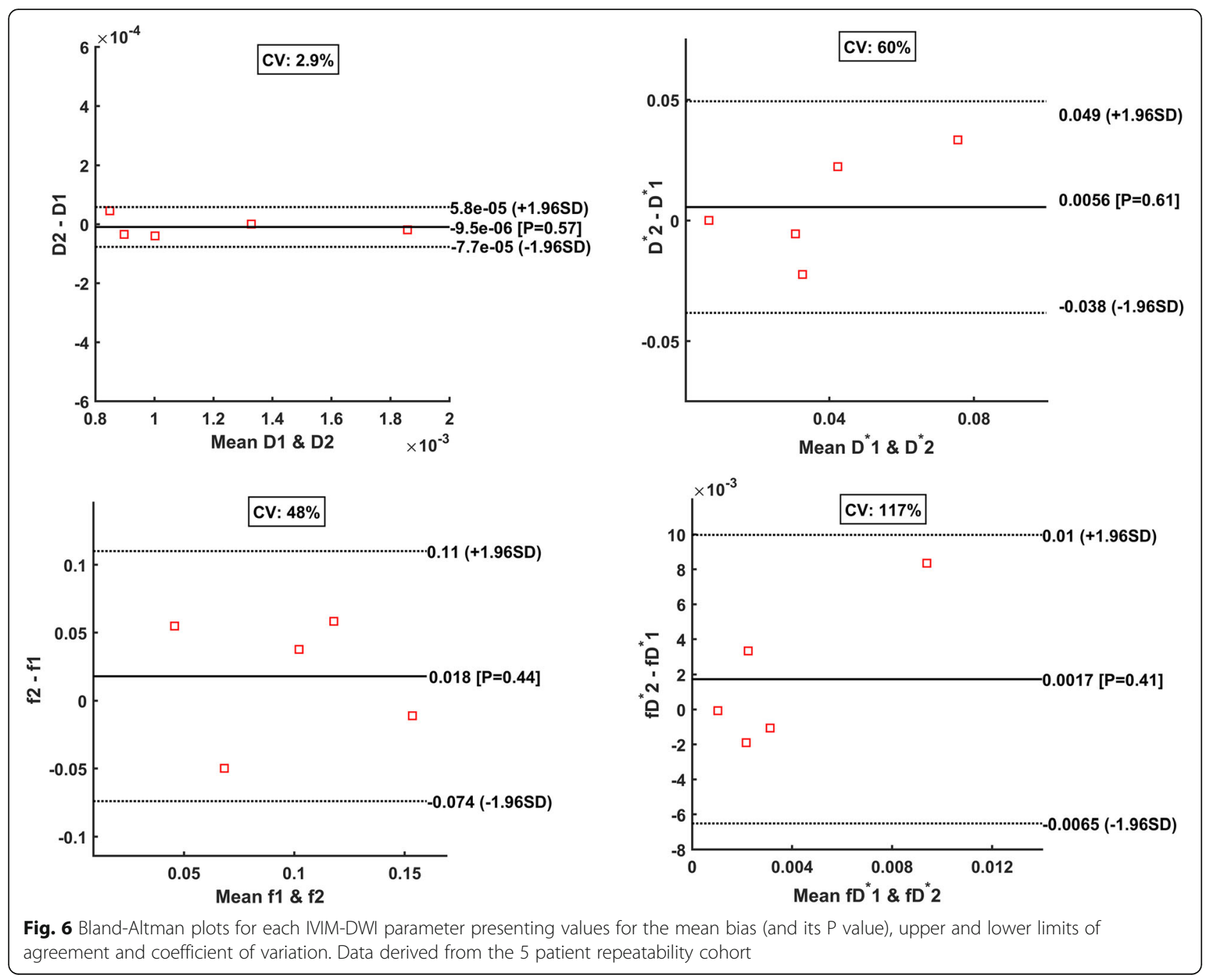

data were motion corrected before method-specific analysis. Both DCE-MRI and IVIM-DWI data were acquired in the coronal plane (where we expect the principle source of motion that affects liver imaging to be superior-inferior in the direction of respiratory motion) and thus allowing our in-plane registration and motion correction to minimize the effects of motion; see further details in the Supplementary material.

Interestingly, the highly significant therapeutic effects observed for DCE-MRI parameters such as $\mathrm{K}^{\text {trans }}$, EF and KEF following Regorafenib treatment were not matched by changes in the perfusion-related IVIM-DWI parameters (f, $\mathrm{D}^{*}$ or $\mathrm{fD}^{*}$; none reached significance). The diffusion coefficient $\mathrm{D}$, a non-perfusion IVIM-DWI parameter, significantly increased by $17 \%$ post treatment $(P<0.001)$, suggesting a reduction in cellularity after treatment. This increase in D was within the capability of our measurement repeatability to confidently detect it. Overall, these results suggest that a multiparametric analysis based on the most sensitive MR parameters (the
3 DCE-MRI parameters and the D coefficient) might yield an improved performance in assessing response to anti-angiogenic treatment.

The highest correlation between DCE-MRI and IVIMDWI methods was moderate $(r=0.67, P<0.001)$ and correlated $\mathrm{K}^{\text {trans }}$ and $\mathrm{fD}^{*}$ parameters, even though changes in $\mathrm{fD}^{*}$ failed to reach statistical significance when measured before and after treatment. The overall results suggest that even though $\mathrm{fD}^{*}$ may be related to $\mathrm{K}^{\text {trans }}$ in these patients, the DCE-MRI measurement is more sensitive than IVIM-DWI for assessing treatment-induced changes in tumour perfusion in colorectal liver metastases. This is further influenced by the fact that perfusion sensitive IVIM-DWI parameters have relatively poor measurement repeatability (i.e. coefficient of variance for $\mathrm{fD}^{*}$ was $\left.117 \%\right)$. Such values were consistent with data derived from larger cohorts reported in the past [13, 24]. This suggests that although IVIM-DWI has been applied for assessment across the body, its role in assessing the vascular properties of hypovascular disease may be 


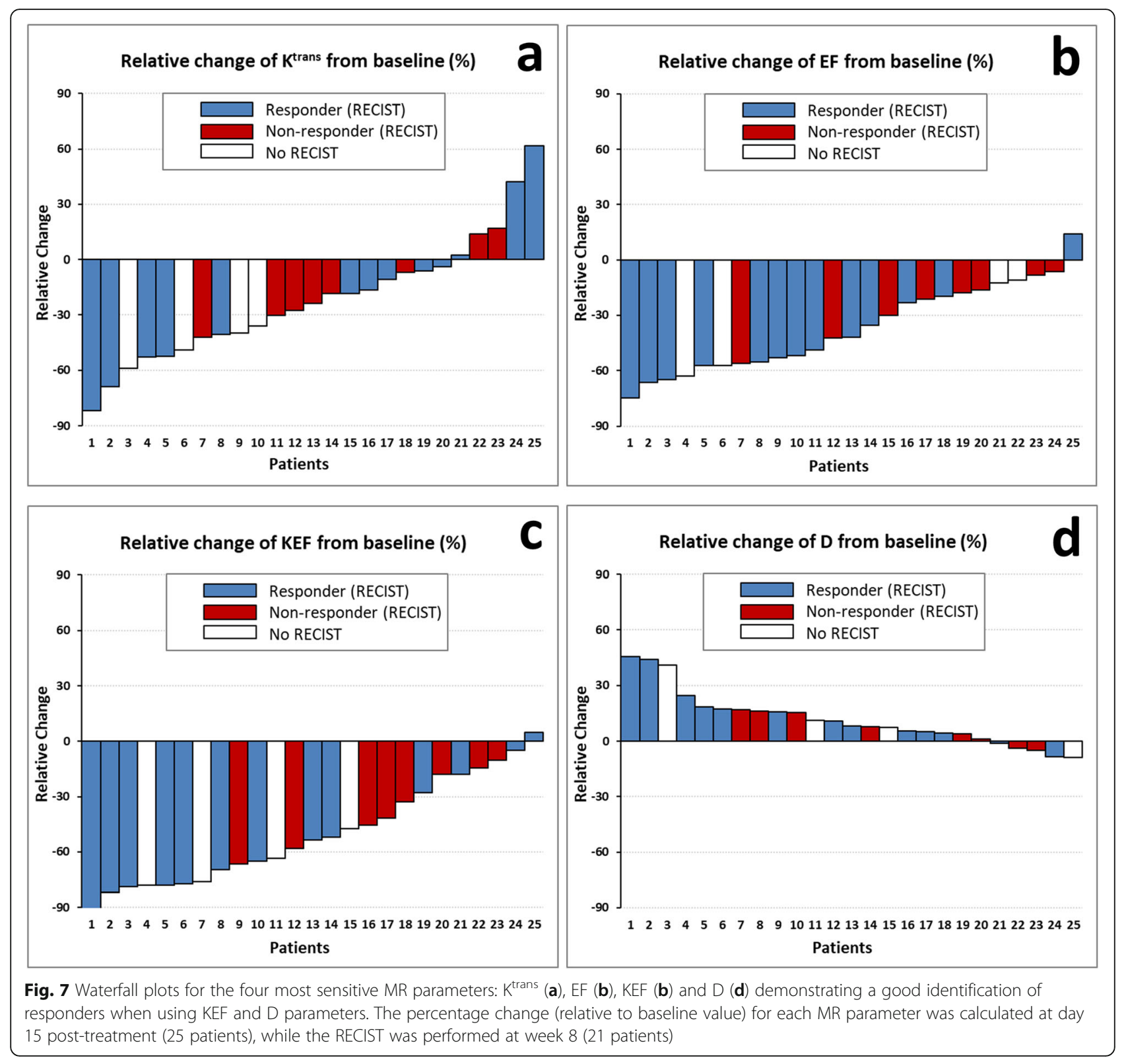

limited due to the combination of diminished sensitivity and poor measurement repeatability.

Another factor to consider when applying IVIM-DWI is that the technique does not measure perfusion in the classical sense [25], even though a link between IVIMDWI parameters and conventional MR perfusion has been hypothesized [26]. However, a few studies in the liver found no correlation between DCE-MRI and IVIMDWI parameters in liver cirrhosis [27] or hepatocellular carcinoma [28]. In another recent study, only a weak correlation was observed between IVIM-DWI and enhancement ratio in hepatocellular carcinoma; nevertheless, DCE-MRI outperformed IVIM-DWI parameters for the identification of necrosis [29].
In terms of clinical translation, DCE-MRI has already been used to demonstrate the pharmacodynamic effects of anti-angiogenic response of other drugs such as Bevacizumb [23, 30] and Sorafenib [31], while the use of IVIMDWI remains limited to exploratory research. Moreover, recommendations towards standardization of DCE-MRI in the liver have been published recently [32], suggesting a wider acceptance of the technique. A similar standardization for IVIM-DWI is not currently available, although [32] includes useful suggestions for DWI standardization as well.

There are a few limitations to our study. First, although we undertook a repeatability study for our IVIM-DWI protocol, we did not perform this for our DCE-MRI 
protocol. The patients in this study were already subjected to a significant number of interventions, and it was difficult to justify undertaking a repeat baseline DCE-MRI study requiring an additional injection of gadolinium contrast. Nevertheless, the liver DCE-MRI protocol was the same as in a previous DCE-MRI study (coronal liver acquisition; 13 patients; population-based arterial input function), where the test-retest repeatability of DCE-MRI was characterised. In that study, a CV of $7.5 \%$ was found for $K^{\text {trans }}$ [33], which corresponds to limits of agreement of $-19 \%,+23 \%$, and we expect similar repeatability values in this study. The change in median $\mathrm{K}^{\text {trans }}$ in our study cohort was $28 \%$, and the individual change in $K^{\text {trans }}$ in $15 / 25$ (i.e. $60 \%$ ) patients was in excess of the previously reported limits of agreement, suggesting a true measured effect.

Second, this was a study in a relatively small population from a single centre. Multi-centre DCE-MRI studies are challenging to perform as there are significant technical and operational challenges in undertaking such studies. Nonetheless, with evolving techniques in acquiring high-temporal resolution and high-spatial resolution images of the liver in free-breathing, it is likely that future DCE-MRI quantification may use semi-automatic or automatic pipelines to allow DCE-MRI parameters to be more widely evaluated in a multi-centre setting. Having said that, in the context of a larger cohort, the perfusion-related IVIM-DWI parameters could reach statistical significance in assessing therapy response.

\section{Conclusions}

In conclusion, in our study of patients with colorectal liver metastases, IVIM-DWI perfusion-related parameters show limited sensitivity to the anti-angiogenic effects of Regorafenib treatment and showed low correlation with DCE-MRI parameters, despite profound and significant post-treatment reductions in DCE-MRI measurements. As such, IVIM-DWI parameters are not currently recommended as an alternative to contrast-based studies when assessing the changes in the vascular properties of colorectal liver metastases in response to treatment with an anti-angiogenic agent.

\begin{abstract}
Abbreviations
DCE-MRI: Dynamic Contrast Enhanced - MRI; IVIM-DWI: Intravoxel Incoherent Motion - Diffusion Weighted Imaging; D: diffusion coefficient; f: perfusion fraction; $D^{*}$ : pseudodiffusion coefficient; $\mathrm{fD}^{*}$ : product of perfusion fraction and pseudodiffusion coefficient; $K^{\text {trans }}$ : volume transfer constant between plasma and extracellular extravascular space; EF: enhancement fraction; $\mathrm{KEF}$ : product of $\mathrm{K}^{\text {trans }}$ and $\mathrm{EF} ; \mathrm{WHO}$ : world health organisation; VIBE: volumetric interpolated breath-hold examination; VOI: volume of interest; R: Spearman correlation coefficient; CV: coefficient of variation; RECIST: Response Evaluation Criteria In Solid Tumors
\end{abstract}

\section{Supplementary information}

The online version contains supplementary material available at https://doi. org/10.1186/s40644-021-00436-0

\section{Additional file 1}

\section{Acknowledgements}

Patients and Staff at the MRI Unit, Royal Marsden Hospital, Sutton, UK. DWI sequence prototype from Thorsten Feiweier, PhD and Berthold Kiefer, PhD Siemens Healthineers, Germany.

\section{Authors' contributions}

MR: study design; acquisition, analysis and interpretation of data; manuscript draft and revision. KK: study design; acquisition and interpretation of data; manuscript revision. DCo: conception and study design; data acquisition and interpretation; manuscript revision. DMK: conception and study design; data acquisition and interpretation; manuscript revision. NT: conception and study design; data acquisition and interpretation; manuscript revision. MAB: conception and study design; data acquisition and interpretation; manuscript revision. JD: software; data analysis; manuscript revision. JW: data interpretation; substantially manuscript revision. SP: data acquisition and analysis. NV: conception and study design; data acquisition and interpretation; manuscript revision. IC: conception and study design; data interpretation; manuscript revision. DC: conception and study design; data interpretation; manuscript revision. MF: conception and study design; data acquisition and interpretation; manuscript revision. MOL: conception and study design; data interpretation; manuscript revision. MRO: study design, software, data analysis and interpretation; manuscript revision. All authors read and approved the final manuscript.

\section{Funding}

CRUK and EPSRC support to the Cancer Imaging Centre at ICR and RMH in association with MRC \& Dept of Health C1060/A10334, C1060/A16464 and NHS funding to the NIHR Biomedical Research Centre and the Clinical Research Facility in Imaging. This report is independent research funded partially by the National Institute for Health Research. The views expressed in this publication are those of the author(s) and not necessarily those of the NHS, the National Institute for Health Research or the Department of Health. MOL was a National Institute for Health Research Emeritus Senior Investigator. IC and DCu would like to acknowledge National Health Service funding to the National Institute for Health Research Biomedical Research Centre at the Royal Marsden NHS Foundation Trust and The Institute of Cancer Research.

\section{Availability of data and materials}

The datasets generated and/or analysed during the current study are not publicly available as appropriate regulatory and institutional approvals are required; datasets might be available from the corresponding author on reasonable request.

\section{Declarations}

Ethics approval and consent to participate

The trial recruitment was approved by our institutional review board (National Research Ethics Service, NRES Committee London-Fulham; Research Ethics Committee reference 14/LO/1812) and informed written consent was obtained from each patient.

\section{Consent for publication}

Not applicable (only anonymised images are included and no individual details are included in the publication).

\section{Competing interests}

MF received honoraria for consulting, advisory role, speaker bureau, and/or research funding from Astellas Pharma, QED Therapeutics, Diaceutics, Tesaro, Roche, Eli Lilly and Novartis.

NV received honoraria from Merck Serono, Pfizer, Bayer and Eli-Lilly. DCum received honoraria from Bayer.

The remaining authors of this manuscript declare that they have no competing interests.

\section{Author details}

'Department of Radiology, MRI Unit, The Royal Marsden NHS Foundation Trust, London, United Kingdom. ${ }^{2}$ Division of Radiotherapy and Imaging, The Institute of Cancer Research, London, United Kingdom. ${ }^{3}$ Department of 
Medicine, Gl and Lymphoma Unit, The Royal Marsden NHS Foundation Trust, London and Sutton, United Kingdom. ${ }^{4}$ Cancer Research UK National Cancer Imaging Translational Accelerator (NCITA), London, United Kingdom. ${ }^{5}$ Centre for Evolution and Cancer, The Institute of Cancer Research, London and Sutton, United Kingdom. ${ }^{6}$ Division of Surgery and Cancer, Faculty of Medicine, Imperial College London, London, United Kingdom. ${ }^{7}$ Department of Medicine (DIMED), Surgical Pathology Unit, University of Padua, Padua, Italy. ${ }^{8}$ Veneto Institute of Oncology IOV-IRCCS, Padua, Italy. ${ }^{9}$ Royal Marsden NHS Foundation Trust \& Institute of Cancer Research, Downs Road, SM2 5PT Sutton, London, UK.

Received: 4 August 2021 Accepted: 24 November 2021

Published online: 19 December 2021

\section{References}

1. Dhillon S. Regorafenib: A Review in Metastatic Colorectal Cancer. Drugs 2018; 78(11): 1133-1144.

2. Krishnamoorthy SK, Relias V, Sebastian S, Jayaraman V, Saif MW. Management of regorafenib-related toxicities: a review. Therap Adv Gastroenterol. 2015;8(5):285-97.

3. Padhani AR, and Husband JE. Dynamic contrast-enhanced MRI studies in oncology with an emphasis on quantification, validation and human studies. Clin Radiol 2001; 56(8): 607-620.

4. Leach MO, Brindle KM, Evelhoch JL, et al. The assessment of antiangiogenic and antivascular therapies in early-stage clinical trials using magnetic resonance imaging: issues and recommendations. Br J Cancer 2005; 92(9): 1599-1610.

5. Leach MO, Morgan B, Tofts PS, et al. Imaging vascular function for early stage clinical trials using dynamic contrast-enhanced magnetic resonance imaging. Eur Radiol 2012; 22(7): 1451-1464.

6. Khan $\mathrm{K}$, Rata M, Cunningham D, et al. Functional imaging and circulating biomarkers of response to regorafenib in treatment-refractory metastatic colorectal cancer patients in a prospective phase II study. Gut 2018; 67(8): 1484-1492.

7. Le Bihan D, Breton E, Lallemand D, Grenier P, Cabanis E, and Laval-Jeantet M. MR imaging of intravoxel incoherent motions: application to diffusion and perfusion in neurologic disorders. Radiology 1986; 161(2): 401-407.

8. Yamada I, Aung W, Himeno Y, Nakagawa T, and Shibuya H. Diffusion coefficients in abdominal organs and hepatic lesions: evaluation with intravoxel incoherent motion echo-planar MR imaging. Radiology 1999; 210(3): 617-623.

9. Koh DM, Collins DJ, and Orton MR. Intravoxel incoherent motion in body diffusion-weighted MRI: reality and challenges. Am J Roentgenol 2011; 196(6): 1351-1361.

10. Zhang H, Li W, Fu C, et al. Comparison of intravoxel incoherent motion imaging, diffusion kurtosis imaging, and conventional DWI in predicting the chemotherapeutic response of colorectal liver metastases. European Journal of Radiology 2020; 130109149.

11. Granata V, Fusco R, Catalano O, et al. Early Assessment of Colorectal Cancer Patients with Liver Metastases Treated with Antiangiogenic Drugs: The Role of Intravoxel Incoherent Motion in Diffusion-Weighted Imaging. PLoS One 2015; 10(11): e0142876.

12. Chiaradia M, Baranes L, Van Nhieu JT, et al. Intravoxel incoherent motion (IVIM) MR imaging of colorectal liver metastases: are we only looking at tumor necrosis? J Magn Reson Imaging 2014; 39(2): 317-325.

13. Andreou A, Koh DM, Collins DJ, et al. Measurement reproducibility of perfusion fraction and pseudodiffusion coefficient derived by intravoxel incoherent motion diffusion-weighted MR imaging in normal liver and metastases. Eur Radiol 2013; 23(2): 428-434.

14. Yang X, Xiao X, Lu B, Chen Y, Wen Z, and Yu S. Perfusion-sensitive parameters of intravoxel incoherent motion MRI in rectal cancer: evaluation of reproducibility and correlation with dynamic contrast-enhanced MRI. Acta Radiol 2019; 60(5): 569-577.

15. Sun $H, X u Y, X u$ Q, et al. Correlation Between Intravoxel Incoherent Motion and Dynamic Contrast-Enhanced Magnetic Resonance Imaging Parameters in Rectal Cancer. Acad Radiol 2019; 26(7): e134-e140.

16. Fram EK, Herfkens $R$, Johnson GA, et al. Rapid calculation of $T 1$ using variable flip angle gradient refocused imaging. Magn Reson Imaging 1987; 5(3): 201-208.

17. Orton MR, Miyazaki K, Koh DM, et al. Optimizing functional parameter accuracy for breath-hold DCE-MRI of liver tumours. Phys Med Biol 2009; 54(7): 2197-2215.

18. Kety SS. The theory and applications of the exchange of inert gas at the lungs and tissues. Pharmacol Rev 1951; 3(1): 1-41.
19. Tofts PS. Modeling tracer kinetics in dynamic Gd-DTPA MR imaging. J Magn Reson Imaging 1997; 7(1): 91-101.

20. Orton MR, d'Arcy JA, Walker-Samuel S, et al. Computationally efficient vascular input function models for quantitative kinetic modelling using DCE-MRI. Phys Med Biol 2008; 53(5): 1225-1239.

21. Parker GJ, Roberts C, Macdonald A, et al. Experimentally-derived functional form for a population-averaged high-temporal-resolution arterial input function for dynamic contrast-enhanced MRI. Magn Reson Med 2006; 56(5): 993-1000.

22. d'Arcy JA, Collins DJ, Padhani AR, Walker-Samuel S, Suckling J, and Leach MO. Informatics in Radiology (infoRAD): Magnetic Resonance Imaging Workbench: analysis and visualization of dynamic contrast-enhanced MR imaging data. Radiographics 2006; 26(2): 621-632.

23. Ferl GZ, O'Connor JPB, Parker GJM, et al. Mixed-effects modeling of clinical DCE-MRI data: Application to colorectal liver metastases treated with bevacizumab. Journal of Magnetic Resonance Imaging 2015; 41(1): 132-141.

24. Orton MR, Messiou C, Collins D, et al. Diffusion-weighted MR imaging of metastatic abdominal and pelvic tumours is sensitive to early changes induced by a VEGF inhibitor using alternative diffusion attenuation models. Eur Radiol 2016; 26(5): 1412-1419.

25. Henkelman RM. Does IVIM measure classical perfusion? Magnetic Resonance in Medicine 1990; 16(3): 470-475.

26. Bihan $\mathrm{DL}$, and Turner R. The capillary network: a link between ivim and classical perfusion. Magnetic Resonance in Medicine 1992; 27(1): 171-178.

27. Patel J, Sigmund EE, Rusinek H, Oei M, Babb JS, and Taouli B. Diagnosis of cirrhosis with intravoxel incoherent motion diffusion MRI and dynamic contrast-enhanced MRI alone and in combination: preliminary experience. J Magn Reson Imaging 2010; 31(3): 589-600.

28. Hectors SJ, Wagner M, Besa C, et al. Intravoxel incoherent motion diffusionweighted imaging of hepatocellular carcinoma: Is there a correlation with flow and perfusion metrics obtained with dynamic contrast-enhanced MRI? J Magn Reson Imaging 2016; 44(4): 856-864.

29. Kakite S, Dyvorne HA, Lee KM, Jajamovich GH, Knight-Greenfield A, and Taouli B. Hepatocellular carcinoma: IVIM diffusion quantification for prediction of tumor necrosis compared to enhancement ratios. Eur J Radiol Open 2016; 31-7.

30. Hirashima $Y$, Yamada $Y$, Tateishi $U$, et al. Pharmacokinetic parameters from 3-Tesla DCE-MRI as surrogate biomarkers of antitumor effects of bevacizumab plus FOLFIRI in colorectal cancer with liver metastasis. Int J Cancer 2012; 130(10):2359-65.

31. Hsu CY, Shen YC, Yu CW, et al. Dynamic contrast-enhanced magnetic resonance imaging biomarkers predict survival and response in hepatocellular carcinoma patients treated with sorafenib and metronomic tegafur/uracil. J Hepatol. 2011 55(4):858-65.

32. Shukla-Dave A, Obuchowski NA, Chenevert TL, et al. Quantitative imaging biomarkers alliance (QIBA) recommendations for improved precision of DWI and DCE-MRI derived biomarkers in multicenter oncology trials. J Magn Reson Imaging 2019; 49(7):e101-e121.

33. Rata M, Collins DJ, Darcy J, et al. Assessment of repeatability and treatment response in early phase clinical trials using DCE-MRI: comparison of parametric analysis using MR- and CT-derived arterial input functions. Eur Radiol 2016; 26(7): 1991-1998.

\section{Publisher's Note}

Springer Nature remains neutral with regard to jurisdictional claims in published maps and institutional affiliations.

\section{Ready to submit your research? Choose BMC and benefit from:}

- fast, convenient online submission

- thorough peer review by experienced researchers in your field

- rapid publication on acceptance

- support for research data, including large and complex data types

- gold Open Access which fosters wider collaboration and increased citations

- maximum visibility for your research: over $100 \mathrm{M}$ website views per year

At $\mathrm{BMC}$, research is always in progress.

Learn more biomedcentral.com/submissions 